\title{
HOT BOTTOM BURNING IN ASYMPTOTIC GIANT BRANCH STARS AND ITS EFFECT ON OXYGEN ISOTOPIC ABUNDANCES
}

\author{
ARNOLD I. BOOTHROYD \\ Canadian Institute for Theoretical Astrophysics, University of Toronto, 60 St. George Street, Toronto, Ontario, Canada M5S 1A7 \\ I.-Juliana SaCKMANN \\ W. K. Kellogg Radiation Laboratory 106-38, California Institute of Technology, Pasadena, CA 91125 \\ AND \\ G. J. WASSERBURG \\ Lunatic Asylum, Charles Arms Laboratory 170-25, California Institute of Technology, Pasadena, CA 91125 \\ Received 1994 October 21; accepted 1995 January 6
}

\begin{abstract}
A self-consistent calculation of asymptotic giant branch (AGB) evolution was carried out, including nucleosynthesis at the base of the convective envelope (hot bottom burning). Hot bottom burning was found to occur for stars between $\sim 4.5$ and $\sim 7 M_{\odot}$, producing envelopes with ${ }^{18} \mathrm{O} /{ }^{16} \mathrm{O} \lesssim 10^{-6}$ and $10^{-3} \lesssim$ ${ }^{17} \mathrm{O} /{ }^{16} \mathrm{O} \lesssim 10^{-1}$. The ${ }^{17} \mathrm{O}$ abundance depends sensitively on the nuclear ${ }^{17} \mathrm{O}$-destruction rate; this rate is only loosely constrained by the requirement that first and second dredge-up models match O-isotope observations of red giant branch (RGB) stars (Boothroyd, Sackmann, \& Wasserburg 1994). In some cases, high mass-loss rates can terminate hot bottom burning before further ${ }^{17} \mathrm{O}$ enrichment takes place or even before all ${ }^{18} \mathrm{O}$ is destroyed. These predictions are in accord with the very limited stellar observations of $\mathrm{J}$ type carbon stars on the AGB and with some of the circumstellar $\mathrm{Al}_{2} \mathrm{O}_{3}$ grains from meteorites. In contrast, precise data from a number of grains and data from most low-mass $\mathrm{S}$ and $\mathrm{C}$ AGB stars $\left(\lesssim 1.7 M_{\odot}\right)$ lie in a region of the ${ }^{18} \mathrm{O} /{ }^{16} \mathrm{O}$ versus ${ }^{17} \mathrm{O} /{ }^{16} \mathrm{O}$ diagram that is not accessible by first and second dredge-up or by hot bottom burning. We conclude that for AGB stars, the standard models of stellar evolution are not in accord with these observations. We surmise that an additional mixing mechanism must exist that transports material from the cool bottom of the stellar convective envelope to a depth at which ${ }^{18} \mathrm{O}$ is destroyed. This "cool bottom processing" mechanism on the AGB is similar to extra mixing mechanisms proposed to explain the excess ${ }^{13} \mathrm{C}$ (and depleted ${ }^{12} \mathrm{C}$ ) observed in the earlier RGB stage of evolution and the large ${ }^{7} \mathrm{Li}$ depletion observed in low-mass main-sequence stars.
\end{abstract}

Subject headings: dust, extinction - nuclear reactions, nucleosynthesis, abundances - stars: abundances stars: AGB and post-AGB

\section{INTRODUCTION}

It has become possible to isolate interstellar oxygen-rich grains from meteorites and to measure their $\mathrm{O}$-isotope ratios with a precision unavailable in typical stellar observations. Each of these grains is considered to be a condensate formed in a stellar outflow, allowing laboratory measurements of isotopic abundance ratios of its parent star. The first three such grains discovered had ${ }^{17} \mathrm{O}$ enrichment factors between 2 and 7 and relatively minor ${ }^{18} \mathrm{O}$ depletions, at most a factor of 1.7 (Huss et al. 1992, 1993, 1994; Nittler et al. 1993; Hutcheon, Huss, \& Wasserburg 1994). In Boothroyd, Sackmann, \& Wasserburg (1994, hereafter BSW-I), we showed that these grains could be accounted for by asymptotic giant branch (AGB) stars that had undergone first (and second) dredge-up (DU), where a deep convective envelope reaches down to the ashes of hydrogen burning. This mixing can result in major ${ }^{17} \mathrm{O}$ enrichments of up to a factor of 20 (depending on stellar mass $M$ ), but only minor ${ }^{18} \mathrm{O}$ depletions (a factor of $\lesssim 1.4$ ). Nittler et al. (1994) recently discovered 21 new interstellar oxide grains, some of which show extreme ${ }^{18} \mathrm{O}$ depletions, up to a factor of 50. It was pointed out by BSW-I that hot bottom burning (HBB) in relatively massive AGB stars $\left(\sim 4-7 M_{\odot}\right)$ could lead to a major destruction of ${ }^{18} \mathrm{O}$. In such stars, the surface convective envelope reaches deep enough that the temperature $T_{\text {ce }}$ at its bottom is high enough for nuclear burning to take place, resulting in $\mathrm{CN}$-cycle processing of the envelope. To provide a sound basis for evaluating the effects of $\mathrm{HBB}$, we carried out extensive AGB calculations, following dozens of $\mathrm{He}$ shell flashes per star. We present some key O-isotope results, comparing them to new grain observations and to recent (less accurate) stellar observations, and discuss the problem of nuclear reaction rates. All previous detailed O-isotope calculations dealt with first and second DU only (Dearborn, Tinsley, \& Schramm 1978; Landré et al. 1990; Dearborn 1992; Schaller et al. 1992; Bressan et al. 1993; El Eid 1994; BSW-I). The present work is a self-consistent extension of the first and second DU work of BSW-I and represents the first detailed calculations of the effect of $\mathrm{HBB}$ on $\mathrm{O}$-isotope ratios.

\section{METHODS AND RESULTS}

We considered stars of $M=3,4,4.5,5,6$, and $7 M_{\odot}$ with solar metallicity $(Z=0.02)$, and $3,4,5$, and $6 M_{\odot}$ with $Z=0.01$ and 0.001 ; in higher mass models, we found that core carbon ignition prevented the AGB stage. Models were evolved self-consistently, starting from the pre-main sequence, following the evolution through first and second DU and through dozens of helium shell flashes (thermal pulses) on the AGB, where third DU and HBB can occur; runs were terminated when numerical problems set in. For details, see Sackmann \& Boothroyd (1992) and Boothroyd, Sackmann, \& 
Ahern (1993). $C / Z=0.2179, N / Z=0.0531$, and $O / Z=0.4816$ (by mass) as in Keady (1985), similar to Ross \& Aller (1976) or Grevesse $(1984)$, and $\left({ }^{16} \mathrm{O} /{ }^{17} \mathrm{O}\right)_{\odot}=2660$ and $\left({ }^{16} \mathrm{O} /{ }^{18} \mathrm{O}\right)_{\odot}=$ 500 (by number). Mass loss was included via wind $M=$ $-\eta\left(4 \times 10^{-13}\right) L R / M$ (where the luminosity $L$, radius $R$, and mass $M$ are in solar units and $\dot{M}$ is in solar masses per year; $\eta$ is the mass loss parameter). For most runs, we used a modest mass-loss rate $(\eta=1.4$; see Kudritzki \& Reimers 1978); as this underestimates the total (observed) AGB mass loss, we also used an intermediate mass-loss rate $(\eta=5$; see de Jong 1983) and a high mass-loss rate $(\eta=14)$, and we tested extreme massloss rates $(\eta=50$ and 140). We used high-temperature opacities from the Los Alamos Opacity Library (LAOL), obtained from Keady (1985). Below $10^{4} \mathrm{~K}$, we generally used molecular opacities from Sharp (1992), but we also tested the effect of older molecular opacities from Keady (1985): the effect on HBB turned out to be small (see also Boothroyd et al. 1993). Both the Sharp and Keady molecular opacities require a value of the convective mixing-length parameter $\alpha=2.1$ in order to match the Sun (Sackmann, Boothroyd, \& Kraemer 1993). Nuclear reaction rates from Caughlan \& Fowler (1988) were used, except for the ${ }^{17} \mathrm{O}$-destruction reactions ${ }^{17} \mathrm{O}(p, \alpha){ }^{14} \mathrm{~N}$ and ${ }^{17} \mathrm{O}(p, \gamma)^{18} \mathrm{~F}\left(e^{+} v\right)^{18} \mathrm{O}$. For these reactions, we generally used the Landré et al. (1990) rates with parameters $f_{1}=0.2$ and $f_{2}=0.1$. The ${ }^{18} \mathrm{O}(p, \alpha)^{15} \mathrm{~N}$ rate, which dominates the destruction of ${ }^{18} \mathrm{O}$, was taken from Caughlan \& Fowler (1988), with the parameter $f=0$. These important rates have not yet been established by laboratory measurements. As a result, the parameters $f_{1}, f_{2}$, and $f$ were obtained by BSW-I by matching first and second DU models with observations of O-isotope ratios in stars on the red giant branch (RGB). For ${ }^{18} \mathrm{O}$ destruction, $f>0$ is excluded by the observations. However, for the ${ }^{17} \mathrm{O}$-destruction rate, $f_{1}$ is not tightly constrained, and $f_{2}$ is not constrained at all (since it affects rates only above $10^{8}$ K). In BSW-I, we chose $f_{1}=0.2$ as the best fit and $f_{2}=0.1$ as an intermediate value; this nuclear parameter set is henceforth referred to as set N1. In this study of more advanced stages of evolution, it was found that the resulting ${ }^{17} \mathrm{O} /{ }^{16} \mathrm{O}$ depended strongly on the choice of $f_{1}$. Therefore, we explored the consequences of choosing $f_{1}=0.5$ or 1 (referred to as set $\mathrm{N} 2$ or N3, respectively); these higher rates give first and second DU results that are still consistent with the RGB observations (though not quite as good a fit). We also explored the consequences of using a different functional form from Landré et al. (1990), namely, the lower ${ }^{17}$ O-destruction rates of Caughlan \& Fowler (1988) with their parameter $f_{1}^{\prime}=1$ (referred to as set N0). For $Z=0.02$ with set $\mathrm{N} 1$ and $\eta=1.4$, the $3 M_{\odot}$ star reached a peak value of $T_{\text {ce }} \sim 7 \times 10^{6} \mathrm{~K}$ at the 30th flash, too low for $\mathrm{HBB}$. The $4 M_{\odot}$ star reached $T_{\text {ce }} \sim 4.5 \times 10^{7} \mathrm{~K}$ when the run was terminated (35th flash), high enough for weak $\mathrm{HBB}$, producing ${ }^{7} \mathrm{Li}$ but not yet affecting $\mathrm{CNO}$ isotopes. The $4.5 M_{\odot}$ and $5 M_{\odot}$ stars reached 6 and $7 \times 10^{7} \mathrm{~K}$, respectively (at the 26th and 17th flashes); vigorous $\mathrm{HBB}$ produced large amounts of ${ }^{7} \mathrm{Li}$ and significant amounts of ${ }^{13} \mathrm{C}$ and destroyed most of the ${ }^{18} \mathrm{O}$. For 6 and $7 M_{\odot}, T_{\text {ce }}$ leveled off at $\sim 10^{8} \mathrm{~K}(55$ and 70 flashes, respectively). As ${ }^{7} \mathrm{Li}$ reached its peak value $\left(\sim 10\right.$ times cosmic), ${ }^{18} \mathrm{O}$ was essentially destroyed $\left(\lesssim 10^{-4}\right.$ solar). Shortly thereafter, ${ }^{13} \mathrm{C} /{ }^{12} \mathrm{C}$ reached 0.3 (the nuclear equilibrium value). Subsequently, ${ }^{7} \mathrm{Li}$ declined steadily, most of the envelope $\mathrm{C}$ was burned to $\mathrm{N}$ (preventing carbon star formation; see Boothroyd et al. 1993), and additional production of ${ }^{17} \mathrm{O}$ (a factor of 3 to 5) took place via ${ }^{16} \mathrm{O}(p, \gamma)$ :

I ${ }^{17} \mathrm{O} /{ }^{16} \mathrm{O} \sim(6-9) \times 10^{-3}$ was attained (note ${ }^{17} \mathrm{O} /{ }^{16} \mathrm{O}_{\odot}=3.8$ $\times 10^{-4}$ ). With $\eta=5$, HBB was prevented for $4 M_{\odot}$ but was not much affected for $M \gtrsim 5 M_{\odot}$. With $\eta=14$, a peak $T_{\text {ce }}$ of only $5 \times 10^{7} \mathrm{~K}$ was reached in the $5 M_{\odot}$ star, yielding only minor depletion of ${ }^{18} \mathrm{O}$. For $M \gtrsim 6 M_{\odot}$, high mass loss caused no significant change in $\mathrm{HBB}$. For $\eta=1.4,5$, and $14, \dot{M}$ reached $\sim 3 \times 10^{-6}, 1 \times 10^{-5}$, and $3 \times 10^{-5} M_{\odot} \mathrm{yr}^{-1}$, respectively, for all stellar masses $(\sim 3$ times that at the onset of flashes). As a rule, decreasing $Z$ mimics the effect of increasing the mass; this also applies to HBB, i.e., $T_{\text {ce }}$ is higher for low $Z$. For the 5 and $6 M_{\odot}$ cases for $Z=0.001$, over 60 flashes were followed. These results were similar to the 6 and $7 M_{\odot}$ cases for $Z=0.02$, yielding large ${ }^{7} \mathrm{Li}$ production, ${ }^{13} \mathrm{C} /{ }^{12} \mathrm{C} \approx 0.3$, complete ${ }^{18} \mathrm{O}$ destruction, and destruction of $\mathrm{C}$ (producing $\mathrm{N}$ ). However, ${ }^{17} \mathrm{O}$ was much more strongly enriched by second DU in the case $Z=0.001\left({ }^{17} \mathrm{O} /{ }^{16} \mathrm{O} \sim 0.009\right.$; as compared with 0.0016 for $Z=0.02$ ). $\mathrm{HBB}$ first reduced ${ }^{17} \mathrm{O} /{ }^{16} \mathrm{O}$ by a factor of 2 , because the nuclear equilibrium abundance was lower than the preflash abundance. As $T_{\text {ce }}$ grows, so does the nuclear equilibrium abundance; at the point where calculations were cut off, ${ }^{17} \mathrm{O} /{ }^{16} \mathrm{O}$ reached 0.009 and 0.013 for the 5 and $6 M_{\odot}$ cases, respectively. Note that after 60 flashes, ${ }^{7} \mathrm{Li}$ had finished its postpeak decline, leveling off at $\log \epsilon\left({ }^{7} \mathrm{Li}\right) \sim-1.5$ (as ${ }^{3} \mathrm{He}$ production balanced ${ }^{3} \mathrm{He}$ destruction); this is on the lower edge of ${ }^{7} \mathrm{Li}$ abundances observed in red giants (Lambert, Dominy, \& Sivertsen 1980; Brown et al. 1989). This result shows that a low level of ${ }^{7} \mathrm{Li}$ does not rule out HBB. After the drop due to $\mathrm{C}$ destruction, $\mathrm{C} / \mathrm{O}$ slowly grew again, as $\mathrm{O}$ burned to $\mathrm{N} ; \mathrm{C} / \mathrm{O}$ would reach unity after another few dozen flashes.

Predicted O-isotope evolution for a number of stellar masses is shown in Figure 1a. Trajectories for first and second DU from BSW-I start at point A, moving in the direction of point B. A slight drop in ${ }^{18} \mathrm{O} /{ }^{16} \mathrm{O}$ is followed by a large increase in ${ }^{17} \mathrm{O} /{ }^{16} \mathrm{O}$; termination of $\mathrm{DU}$ is indicated by the labeled open squares (before this point, mass-loss rates are low, and few grains are formed). For $4.5 M_{\odot} \lesssim M \lesssim 7 M_{\odot}$ HBB occurs, causing a departure from $\mathrm{AB}$ at the point corresponding to second DU. The trajectory falls nearly vertically, with destruction of ${ }^{18} \mathrm{O}$; this occurs quite rapidly for $M>5 M_{\odot}$. For $M=6$ and $7 M_{\odot}$ there is subsequently a much slower increase of ${ }^{17} \mathrm{O}$, on a timescale that can be comparable to the AGB lifetime; large ${ }^{17} \mathrm{O} /{ }^{16} \mathrm{O}$ values are eventually attained. High mass-loss rates can prevent ${ }^{18} \mathrm{O}$ destruction for $M \lesssim 5 M_{\odot}$ and reduce slightly the ${ }^{17} \mathrm{O}$ enrichment for $M \gtrsim 6 M_{\odot}$ (the endpoint for $5 M_{\odot}$ with $\eta=14$ is shown by the solid square in Fig. $1 a$ ). Figure $1 a$ also shows the results of using higher ${ }^{17} \mathrm{O}$ destruction rates (sets N2 and N3). Note that in this case first and second DU follow essentially curve AB but stop at somewhat lower ${ }^{17} \mathrm{O} /{ }^{16} \mathrm{O}$. Subsequent evolution is similar to case $\mathrm{N} 1$, except that the ${ }^{17} \mathrm{O}$ enhancement is greatly reduced; for case $\mathrm{N} 3$, the maximum value of ${ }^{17} \mathrm{O} /{ }^{16} \mathrm{O}$ hardly exceeds the value at second DU. For $M \gtrsim 6 M_{\odot},{ }^{18} \mathrm{O} /{ }^{16} \mathrm{O} \approx 0$; mass-loss rates extreme enough $(50<\eta<140)$ to prevent complete ${ }^{18} \mathrm{O}$ destruction seem improbable, as they would also prevent the stars from reaching the high luminosities $\left(M_{\text {bol }} \sim-7\right)$ observed in some Magellanic Cloud AGB stars undergoing HBB (Smith \& Lambert 1989, 1990). We cannot identify any combination of parameters that would result in low ${ }^{18} \mathrm{O} /{ }^{16} \mathrm{O}$ combined with ${ }^{17} \mathrm{O} /{ }^{16} \mathrm{O}<0.001$ or $\gtrsim 0.005$ : the hatched regions in Figure $1 a$ thus appear to be inaccessible by $H B B$.

\section{COMPARISON WITH GRAIN AND STELLAR OBSERVATIONS}

The curves for first and second DU and for HBB are combined with the observational data in Figure $1 b$. Stellar observations have large uncertainties, but most data lie on or below curve AB. Most observations of normal $\mathrm{S}$ and $\mathrm{C}$ stars have 


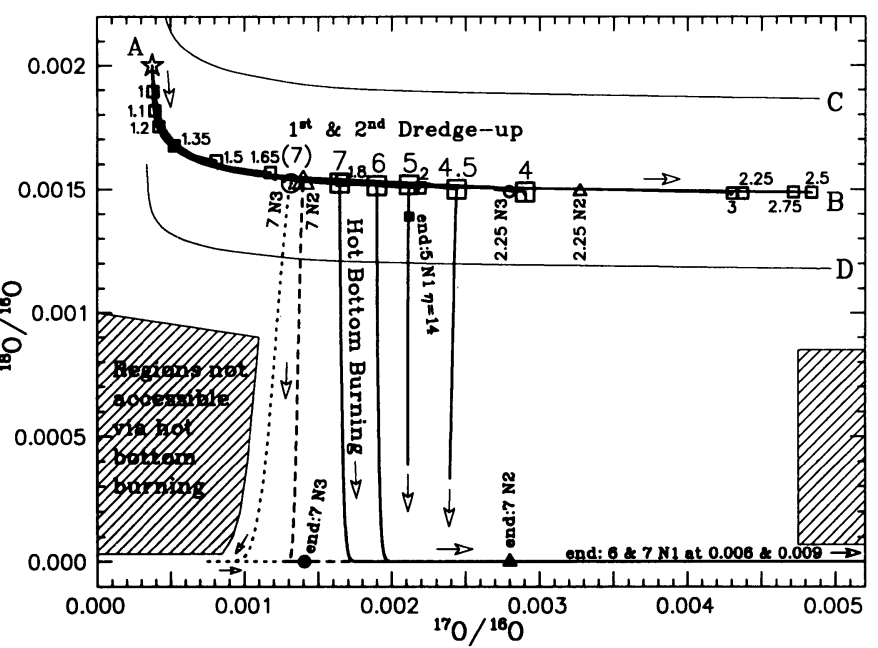

FIG. $1 a$

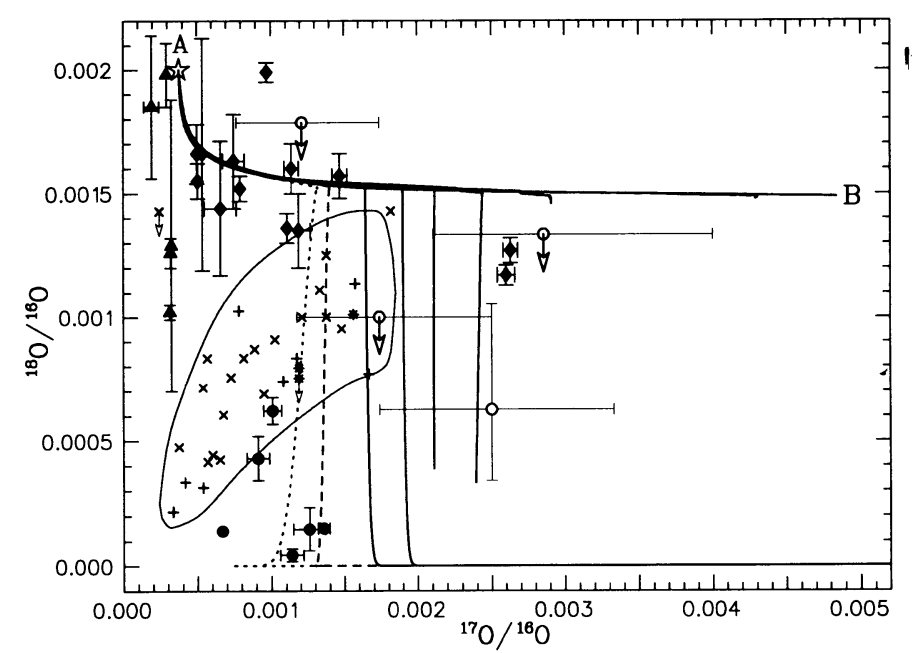

Fig. $1 b$

Fig. 1.-(a) Predicted evolution of ${ }^{18} \mathrm{O} /{ }^{16} \mathrm{O}$ vs. ${ }^{17} \mathrm{O} /{ }^{16} \mathrm{O}$ for first and second dredge-up (DU) and hot bottom burning (HBB) in $Z=0.02$ stars of masses from 1 to $7 M_{\odot}$. Initial ratios were solar (point $\mathrm{A}$ ); curves $\mathrm{C}$ and $\mathrm{D}$ indicate the effect of varying initial ${ }^{18} \mathrm{O} /{ }^{16} \mathrm{O}$ by $\pm 20 \%$ and ${ }^{17} \mathrm{O} /{ }^{16} \mathrm{O}$ by $\pm 10 \%$. Heavy solid curves refer to nuclear rate set N1; termination of first DU $\left(M \leq 3 M_{\odot}\right)$ or second DU $\left(M \geq 4 M_{\odot}\right)$ is indicated by small or large open squares, respectively, labeled by the stellar mass. Dashed and dotted curves refer to the higher ${ }^{17} \mathrm{O}$-destruction rates ( $\mathrm{N} 2$ and $\mathrm{N} 3$ ), with the end of first or second DU indicated by open triangles or open circles (for 2.25 and $7 M_{\odot}$ ). HBB first decreases ${ }^{18} \mathrm{O}$ sharply from its second DU value (heavy vertical lines) and, then increases the ${ }^{17} \mathrm{O}$ abundance. Filled squares, triangles, and circles show cases where $\mathrm{O}$-isotope evolution terminates owing to either mass loss turning off $\mathrm{HBB}$ or nuclear equilibrium ratios being attained. Hatched regions are inaccessible to either first or second DU or HBB, for all initial states considered. (b) Comparison of model with grain and stellar observations. Of four $\mathbf{J}$ type carbon stars (open circles), with ${ }^{13} \mathrm{C} /{ }^{12} \mathrm{C} \sim 0.3$ indicating $\mathrm{HBB}$, three have only upper limits (heavy arrows) for ${ }^{18} \mathrm{O}$. Normal $\mathrm{S}$ stars (plus signs) and $\mathrm{C}$ stars (crosses) on the AGB have large errors (not shown), comparable to those of the J star observations; circumstellar C star observations (asterisk) of Kahane et al. (1992) are somewhat more accurate. High-precision grain data are shown by filled symbols, grouped as by Nittler et al. (1994): group 1 (diamonds) is consistent with first and second DU, group 2 ( filled circles) displays very $l w^{18} \mathrm{O} /{ }^{16} \mathrm{O}$ (requiring ${ }^{18} \mathrm{O}$ destruction), and group 3 (triangles at left) displays low ${ }^{17} \mathrm{O} /{ }^{16} \mathrm{O}$.

uncertainties (not shown) of order $50 \%$ (Harris, Lambert, \& Smith 1985; Harris et al. 1987; Kahane et al. 1992). These are AGB stars with $\mathrm{C} / \mathrm{O} \gtrsim 1$ that have undergone third $\mathrm{DU}$ (mixing $s$-process elements and ${ }^{12} \mathrm{C}$ to the surface). Their ${ }^{13} \mathrm{C} /{ }^{12} \mathrm{C}$ ratios are considerably below the nuclear equilibrium value of $\sim 0.3$, indicating that strong $\mathrm{HBB}$ cannot be taking place. The O-isotopes of these stars cannot be accounted for by first, second, and third DU or by weak $\mathrm{HBB}$, since many have ${ }^{17} \mathrm{O}$ abundances that are too low for such an explanation. These stars are likely to be common low-mass AGB stars ( $M<2 M_{\odot}$ ), with ${ }^{17} \mathrm{O}$ resulting from first DU but with additional ${ }^{18} \mathrm{O}$ depletion. The four open circles in Figure $1 b$ indicate stellar observations for AGB stars where the presence of strong $\mathrm{HBB}$ is suggested by ${ }^{13} \mathrm{C} /{ }^{12} \mathrm{C} \sim 0.3$ (Harris et al. 1987). Within the huge uncertainties, these stellar O-isotope ratios are consistent with our models of $\mathrm{HBB}$, but the data certainly are not diagnostic. High-precision grain measurements (see Fig. 1b) can be divided into three groups (Nittler et al. 1994). Group 1 grains can be understood in terms of first and second DU, with slight variation of initial O-isotope ratios (BSW-I). The six group 2 grains stand out, in that they have very low ${ }^{18} \mathrm{O} /{ }^{16} \mathrm{O}$ ratios, which cannot be the result of first and second DU. Three of these grains could possibly be interpreted as products of $\mathrm{HBB}$, provided that they originated from $\sim 7 M_{\odot}$ stars and that the ${ }^{17} \mathrm{O}$-destruction nuclear rate was very high (set N2 or N3). In addition, improbably high mass-loss rates would be required to explain the observed incomplete ${ }^{18} \mathrm{O}$ destruction, although it is also possible that some of the ${ }^{18} \mathrm{O}$ measured in these grains was the result of oxygen contamination during ion probe analysis. On the other hand, the other three group 2 grains lie in the region inaccessible to $\mathrm{HBB}$, near the low-mass C and S AGB star observations. Group 3 grains exhibit ${ }^{17} \mathrm{O} /{ }^{16} \mathrm{O}$ ratios lower than solar; Nittler et al. (1994) suggest that these might be produced in massive stars. However, calculations of Weaver \& Woosley (1993) suggest that on average, the opposite is the case in the ejected material $\left({ }^{17} \mathrm{O} /{ }^{16} \mathrm{O} \gtrsim\right.$ solar $)$. Four of the five group 3 grains might actually originate in low-mass stars with initial stellar ${ }^{17} \mathrm{O} /{ }^{16} \mathrm{O}$ ratios slightly below solar: one grain lies in the region of group 1 , and three lie in a region between groups 1 and 2 .

\section{COOL BOTTOM PROCESSING}

It is clear that some of the group 2 grains, as well as many of the stellar observations, cannot be explained by HBB or by first and second DU from the stellar models. The AGB stars in the "inaccessible region" are of low mass $\left(M<2 M_{\odot}\right)$, and their envelopes have undergone CNO cycle processing (as shown by the ${ }^{18} \mathrm{O}$ depletion). This suggests that some "extra mixing" mechanism exists that transports material from the cool bottom of the convective envelope to sufficient depths that ${ }^{18} \mathrm{O}$ is destroyed (i.e., $T \gtrsim 2 \times 10^{7} \mathrm{~K}$ ); we call this "cool bottom processing." Such a mechanism has been proposed to explain anomalous observations of other isotopes. For example, during the red giant branch (RGB) phase, shortly subsequent to first DU, many low-mass stars have been observed to acquire additional ${ }^{13} \mathrm{C}$ enrichment, beyond what was produced by first DU (see, e.g., Gilroy \& Brown 1991). In low-mass, low- $Z$ RGB stars, a pronounced decline in $\mathrm{C}$ is observed (see Smith \& Tout 1992). On the main sequence, large ${ }^{7} \mathrm{Li}$ depletions are observed in low-mass stars. The above abundance anomalies all point to extra nuclear processing of envelope material, at temperatures higher than reached at the bottom of the convective envelopes. It has been frequently pointed out that some slow or episodic extra mixing mechanism must exist that transports material from the cool convective envelope down to regions hot enough for nuclear 
processing. Other observations indicate that this mixing cannot be ordinary convection: e.g., helioseismological measurements of the depth of solar convection and observations of the presence of lithium in ${ }^{13} \mathrm{C}$-enhanced RGB stars (Wallerstein \& Morell 1994). The extent and effect of this extra mixing depends on the stage of evolution as well as on the star's mass. Effects attributed to such extra mixing have been observed only in relatively low mas stars $\left(M \lesssim 2 M_{\odot}\right)$, and the effects seem to be largest for the lowest masses. Although anomalous ${ }^{18} \mathrm{O}$ depletion is observed to have occurred in AGB stars, no such depletion has yet been observed in RGB stars; however, more ${ }^{18} \mathrm{O}$ observations are needed on the RGB, especially in ${ }^{13} \mathrm{C}$-enriched stars. It is clear that further progress in understanding the abundances of oxygen isotopes will depend on laboratory measurements of the cross sections.

\section{ENRICHMENT OF THE INTERSTELLAR MEDIUM}

The ${ }^{17} \mathrm{O}$ enrichment of the interstellar medium has three sources. Supernovae are a key source (Weaver \& Woosley
1993); comparable amounts can be produced in low-mass stars $\left(M \lesssim 4 M_{\odot}\right.$ : BSW-I). For $5 \lesssim M \lesssim 7 M_{\odot}$, HBB may produce significant amounts of ${ }^{17} \mathrm{O}$, but the amount is much more sensitive to the ${ }^{17} \mathrm{O}$ destruction rate than in the other two sources. Thus, HBB may be responsible for anywhere between $5 \%$ (set N3) and twice as much (set N0) ${ }^{17} \mathrm{O}$ production as the other two sources combined. HBB stars process too little of the interstellar medium to significantly reduce its ${ }^{18} \mathrm{O}$ abundance.

We appreciate the support supplied by the Kellogg Radiation Laboratory. A. I. B. thanks S. D. Tremaine and P. G. Martin for the support provided by the Canadian Institute for Theoretical Astrophysics. I.-J. S. wishes to thank R. F. Christy for challenging discussions and gentlemanly help during the many tasks of daily life. This work was supported by grants from the National Science Foundation PHY-8817296, the Natural Sciences and Engineering Research Council of Canada, and NASA grants NAGW-3040, NAGW-3337, and NAGW-3297. Caltech Division Contribution 5464(858).
Boothroyd, A. I., Sackmann, I.-J., \& Ahern, S. C. 1993, ApJ, 416, 762 Boothroyd, A. I., Sackmann, I.-J., \& Wasserburg, G. J. 1994, ApJ, 430, L77 (BSW-I)

Bressan, F., Fagotto, G., Bertelli, G., \& Chiosi, C. 1993, A\&AS, 100, 674

Brown, J. A., Sneden, C., Lambert, D. L., \& Dutchover, E., Jr. 1989, ApJS, 71, 293

Caughlan, G. R., \& Fowler, W. A. 1988, Atomic Data Nucl. Data Tables, 40, 205

Dearborn, D. S. P. 1992, Phys. Rep., 210, 367

Dearborn, D., Tinsley, B. M., \& Schramm, D. N. 1978, ApJ, 223, 557

de Jong, T. 1983, ApJ, 274, 252

El Eid, M. F. 1994, A\&A, 285, 915

Gilroy, K. K., \& Brown, J. A. 1991, ApJ, 371, 578

Grevesse, N. 1984, Phys. Scripta, T8, 49

Harris, M. J., Lambert, D. L., Hinkle, K. H., Gustafsson, B., \& Eriksson, K. 1987, ApJ, 316, 294

Harris, M. J., Lambert, D. L. \& Smith, V. V. 1985, ApJ, 299, 375

Huss, G. R., Fahey, A. J., Gallino, R., \& Wasserburg, G. J. 1994, 430, L81

Huss, G. R., Hutcheon, I. D., Fahey, A. J., \& Wasserburg, G. J. 1993, Meteoritics, 28,369

Huss, G. R., Hutcheon, I. D., Wasserburg, G. J., \& Stone, J. 1992, Lunar

Planet. Sci., 23, 563

\section{REFERENCES}

Hutcheon, I. D., Huss, G. R., \& Wasserburg, G. J. 1994, ApJ, 425, L97

Kahane, C., Cernicharo, J., Gómez-González, J., \& Guélin, M. 1992, A\&A, 256,235

Keady, J. 1985, private communication

Kudritzki, R. P., \& Reimers, D. 1978, A\&A, 70, 227

Lambert, D. L., Dominy, J. F., \& Sivertsen, S. 1980, ApJ, 235, 114

Landré, V., Prantzos, N., Auger, P., Bogaert, G., Lefebvre, A., \& Thibaud, J. P. 1990, A\&A, 240, 85

Nittler, L. R., Alexander, C. M. O., Gao, X., Walker, R. M., \& Zinner, E. K. 1994, Nature, 370, 443

Nittler, L. R., Walker, R. M., Zinner, E., Hoppe, P., \& Lewis, R. S. 1993, Lunar Planet. Sci., 24, 1087

Ross, J. E., \& Aller, L. H. 1976, Science, 191, 1223

Sackmann, I.-J., \& Boothroyd, A. I. 1992, ApJ, 392, L71

Sackmann, I.-J., Boothroyd, A. I., \& Kraemer, K. E. 1993, ApJ, 418, 457

Schaller, G., Schaerer, D., Meynet, G., \& Maeder, A. 1992, A\&AS, 96, 269

Sharp, C. M. 1992, A\&AS, 94, 1

Smith, G. H., \& Tout, C. A. 1992, MNRAS, 256, 449

Smith, V. V., \& Lambert, D. L. 1989, ApJ, 345, L75

. 1990, ApJ, 361, L69

Wallerstein, G., \& Morell, O. 1994, A\&A, 281, L37

Weaver, T. A., \& Woosley, S. E. 1993, Phys. Rep., 227, 65 